\title{
Recent developments in durability mesomechanics of concrete, including cracking via interface elements
}

\author{
Joaquin Liaudat, Mariana Rodriguez, Carlos Lopez and Ignacio Carol \\ Department of Geotechnical Engineering and Geo-Sciences \\ ETSECCPB (School of Civil Engineering)-UPC (Technical Univ. of Catalonia) \\ 08034 Barcelona \\ e-mail: joaquin.liaudat@upc.edu,mariana.rodriguez@upc.edu, \\ carlos.maria.lopez@upc.edu, ignacio.carol@upc.edu.
}

\begin{abstract}
The mesomechanical model for concrete previously developed for purely mechanical actions, is used as the basis for coupled durability mechanics calculations. Until now, the phenomena studied include drying shrinkage, external sulfate attack and mechanical effects of high temperatures. On-going work is focusing on alkali-silica reaction (ASR). From the chemical viewpoint, ASR is represented with a three-field diffusion-reaction chemical model, which is shown to qualitatively and quantitatively reproduce some experimental measurements of sandwich-type specimens subject to standardized accelerated ASR tests. The model developed includes the recently identified role of Calcium in this type of expansive reactions.
\end{abstract}

\section{INTRODUCTION}

Constantly increasing computer power is fostering the development of sophisticated material degradation models including phenomena such as humidity, temperature and/or chemical processes coupled with mechanical behavior. On the other hand, micro/meso-level analysis of heterogeneous and quasi-brittle materials such as concrete or rock has emerged as a powerful tool for the modeling of the cracking and fracture processes under different loading situations. During over a decade, the group of Mechanics of Materials at UPC (Barcelona) has been developing such type of combined tools with the distinctive feature of representing cracks via zero-thickness interface elements, for progressively more complex loading/environmental scenarios. The approach was first applied to the purely mechanical behavior in 2D (López et al, 2000, 2008) and 3D (Caballero et al, 2007), then to basic creep (López,et al, 2001)., drying shrinkage (Idiart et al, 2011a) and external sulfate attack (Idiart et al, 2011b). In this paper, the latest of those applications are briefly summarized, and the most recent developments are described in more detail: the application to the mechanical effects of high temperatures, and the on-going work on alkali-silica reaction. 


\section{MESOMECHANICAL MODEL}

The models developed for coupled durability phenomena are based on an underlying meso-mechanical model in which the largest aggregate particles are represented explicitly, surrounded by a homogeneous matrix representing the average behavior of mortar plus the smaller aggregates. In order to capture the main potential crack trajectories, zero-thickness interface elements are inserted a priori of the analysis, and are equipped with a nonlinear constitutive law based on elasto-plasticity with concepts of fracture mechanics (Carol et al., 1997; Carol et al., 2001). Results of the meso-mechanical model for normal concrete specimens subject to a variety of loading cases in 2D and 3D can be found elsewhere (Carol et al., 2001; Caballero et al., 2007; López et al., 2008).

\section{HYGRO- AND CHEMO-MECHANICAL PROBLEMS}

Transport model and coupling strategy. The diffusion analysis of moisture or ionic species in the concrete is based on Fick's second law, with a variable coefficient of diffusivity. The diffusion analysis is combined efficiently with the mechanical analysis by using the same mesh for both. This requires interface elements with double nodes also for the diffusion analysis, as proposed by Segura and Carol (2004). Such interface elements for diffusion incorporate longitudinal as well as transversal diffusivities $K_{l}$ and $K_{t}$. In the absence of specific information, the latter may be given a very high value, while $K_{l}$ is assumed to increase with a law proportional to the cube or square of the crack opening (Idiart et al., 2011a). The diffusion of moisture or ions drives chemical and/or physical processes that lead to volume changes in the cement paste, which in general are not uniform and therefore lead to stresses and possibly cracks. These cracks are the vehicle for coupling, ie. they represent an easier way for moisture or ionic species to move through the specimen, thus accelerating the volume changes and therefore increasing cracking again. The coupling is implemented via staggered strategy for each time step of the time discretization.

Drying shrinkage. Drying shrinkage is phenomenon driven by the loss of moisture, of the initially saturated concrete, to the environment. In the model developed, the moisture movement is represented by a diffusion equation in terms of relative humidity in the pores, $H$ (Eqn. 1a), in which the diffusivity coefficient is not constant (Eqn. 1b). Once this equation solved with the appropriate boundary conditions, the water loss $\omega_{e}$ (mass of water per unit volume of concrete) can be calculated as a postprocessing of the resulting $H$, by using a desorption isotherm curve. Water loss causes volume reductions $\varepsilon_{s h}$ in the cement paste, which as a first approach may be assumed linear (Eqn. 1c). Further details of the shrinkage model and examples of application may be found in (Idiart et al., 2011a).

$$
\frac{\partial H}{\partial t}=\frac{\partial}{\partial x}\left(D_{H} \frac{\partial H}{\partial x}\right) ; \quad D_{H}(H)=D_{0}+\left(D_{1}-D_{0}\right) f(\beta, H) ; \quad \varepsilon_{s h}=\alpha\left(\omega_{e}\right) \cdot \omega_{e}
$$

External sulfate attack. This degradation phenomenon is originated by the ingress of sulfate ions from the environment into the cement pores. These ions then react 
with solid ingredients of the cement paste leading to volume expansion. It seems accepted that the reaction takes place in two steps: first, sulfate ions react with the portlandite and/or calcium silicate hydrates of the cement paste leading to gypsum, and second, gypsum reacts with various kinds of calcium aluminates leading to formation of secondary ettringite, which is really the expansive product. In the model developed, this mechanism is represented via equation (2), which is mathematically similar to (1) except for the extra sink term which corresponds to the consumption of sulfate ions. Isotropic expansion of the cement paste due to secondary ettringite precipitation is obtained from equation (3), as the product of the total aluminate reacted $C_{e q}^{\text {react }}$ by an equivalent molar volume $\alpha_{s}$. The second term in the equation accounts for the volume of capillary pores $f \Phi_{\text {ini }}$ that has to be filled before any macroscopic expansion is observed.

$$
\begin{gathered}
\frac{\partial U}{\partial t}=\frac{\partial}{\partial x}\left(D_{U} \frac{\partial U}{\partial x}\right)-k U C ; D_{U}(H)=D_{0}+\left(D_{1}-D_{0}\right) f\left(\beta, \Phi_{c a p}\right) \\
\varepsilon_{v}(t)=\max \left|\alpha_{s} \cdot C_{e q}^{r e a c t}-f \Phi_{\mathrm{ini}}, 0\right|
\end{gathered}
$$

A simple application example of the model is shown in Figure 1. A square concrete specimen is subject to sulfate attack from its perimeter, under both coupled (top diagrams of the figure) and uncoupled analysis (bottom diagrams). In the uncoupled analysis, diffusion is unaffected by cracking, while in the coupled analysis cracks work as penetration channels for sulfate ions. Note that, in the coupled case, the emerging perimetral crack eventually becomes a new attack front, leading to the total penetration of the sulfates, while in the uncoupled analysis penetration is limited to a layer near the surface. Further details of the formulation and examples of application may be found in Idiart et al. (2011b).

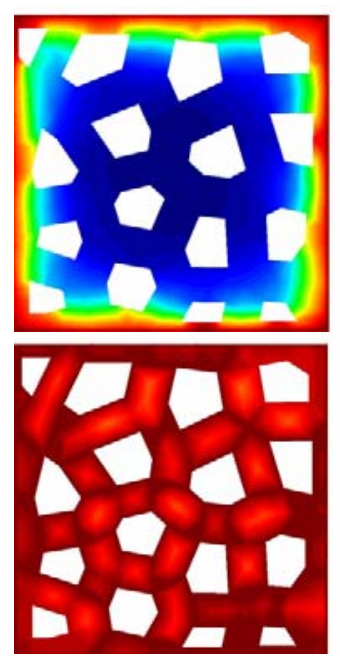

Sulfate concentration

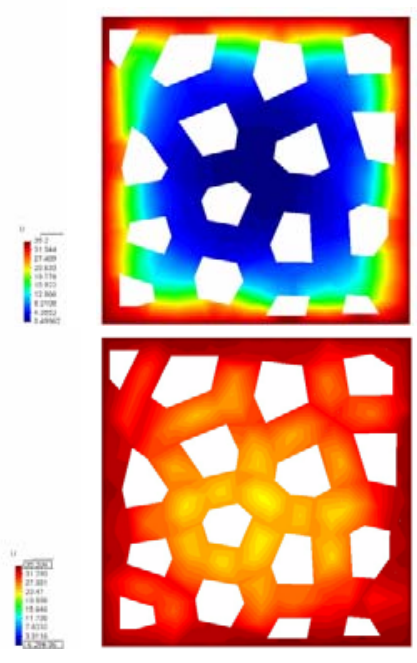

Ettringite Concentration

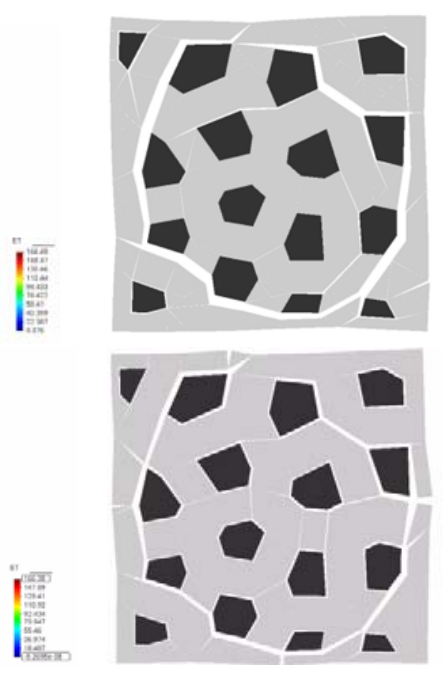

Mechanical response: expansion, cracking and spalling

Figure 1: Simulations at the meso-level of external sulfate attack to concrete. Final stage: 4,600days; high C3A content: 9,13\%. Uncoupled (first row) and coupled analysis (second row). 
Alkali Silica Reaction. The reaction mechanism proposed involves three coupled diffusion processes, two in-going and one out-going from the aggregate viewpoint. Alkali $\left(\mathrm{Na}^{+}\right.$and $\left.\mathrm{K}^{+}\right)$and Calcium $\left(\mathrm{Ca}^{2+}\right)$ ions diffuse "inwards", from high molar concentration sites in the pores of the cement paste phase of the concrete specimen or at its boundaries, towards the aggregate-cement paste interfaces or the inner cracks of the aggregates. The $\mathrm{OH}^{-}$ions associated with alkali and calcium ions attack certain forms of silica in the aggregates (the "reactive silica"), dissolving it in the form of silicate ions which in turn diffuse back to the cement paste phase ("outwards"). The final potentially deleterious ASR precipitation process involves those silicate ions, plus calcium and alkalis. It takes place wherever the reactants are available by precipitating Calcium-Alkali-Silicate-Hydrates (CASH) with a molar volume depending on the reactants concentrations. These diffusion- reaction processes are summarized in the following system of equations (4) where $a, c$ and $s$ are the molar concentrations of alkali, calcium and silicate ions respectively. The source/sink terms are given by chemical kinetics equations (reactive silica dissolution, CASH formation) and chemical equilibrium equations (water self-ionization and portlandite dissociation).

$$
\frac{\partial a}{\partial t}=\frac{\partial}{\partial x}\left(D^{a} \frac{\partial a}{\partial x}\right)+\dot{a} ; \frac{\partial c}{\partial t}=\frac{\partial}{\partial x}\left(D^{c} \frac{\partial c}{\partial x}\right)+\dot{c} ; \frac{\partial s}{\partial t}=\frac{\partial}{\partial x}\left(D^{s} \frac{\partial s}{\partial x}\right)+\dot{s}
$$

During the development of the reaction the volume fractions of the solid components in the material are not constant. Portlandite and reactive silica are progressively dissolved reducing their volumes and increasing the capillary porosity of the material. The CASH formed occupies this empty volume, leading to a net volumetric expansion when the volume of CASH produced exceeds the available pore space. The volume fraction $u_{k}(x)$ corresponding to the solid component $k$ is calculated by means of the molar volume and concentration of the chemical species associated to the component, so that the total volume $V(x)$ is given by equation (5a). Then, the net isotropic expansion due to ASR is obtained from equation (6). The volumetric distribution of the solid components at each location determines the overall diffusion coefficients by equation (6) ( similar equations are considered for $D^{a}(x)$ and $\left.D^{c}(x)\right)$ where $d_{k}$ is the diffusion coefficient of the solid phase $k$, and $m_{s}$ is a coefficients that introduces the effect of the ion type (electric charge, ionic radius). Further details of the formulation may be found in Liaudat et al. (2013).

$$
\begin{gathered}
V(x)=\sum_{k=1}^{8} u_{k}(x) \quad ; \quad D^{s}(x)=\frac{m_{s}}{V(x)} \sum_{k=1}^{8} u_{k}(x) \cdot d_{k} \\
\varepsilon_{A S R}(x)=V(x)-1 \quad \text { if } V(x)>1 \text { (and } 0 \text { otherwise) }
\end{gathered}
$$

An example of application in 1D which simulates an ongoing experimental test is presented to illustrate the capabilities of the model to reproduce realistically the ASR process. The experimental test has been set up to measure ASR expansions at the level of single interface between the hardened cement paste (HCP) and aggregate. Cylindrical "sandwich" specimens of $33 \mathrm{~mm}$ of diameter and $64 \mathrm{~mm}$ of total height, with a borosilicate glass disc in the middle as reactive aggregate, and HCP on top and bottom, are exposed to a $1 \mathrm{M} \mathrm{NaOH}$ solution at $60^{\circ} \mathrm{C}$ for a period of 14 days during which expansions are measured regularly. 
The overall expansion curve obtained with the model is plotted together with the experimental results in Figure 2. The main trend of experimental behavior is caught by the model. The volumetric solid phase distribution in the area near the HCP-glass interface, for different exposure times, is represented in Figure 3. There, the progressive glass and portlandite depletion near the interface becomes apparent, as well as the CASH precipitation, first filling the capillary pores and finally producing a volumetric expansion.

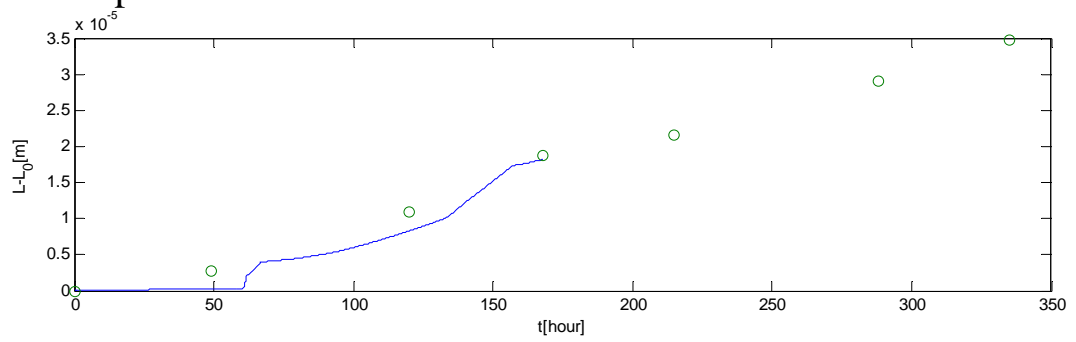

Figure 2: Experimental ASR expansion test at the level of a single aggregateHCP interface. Length change from experiment (circles) and model (line).
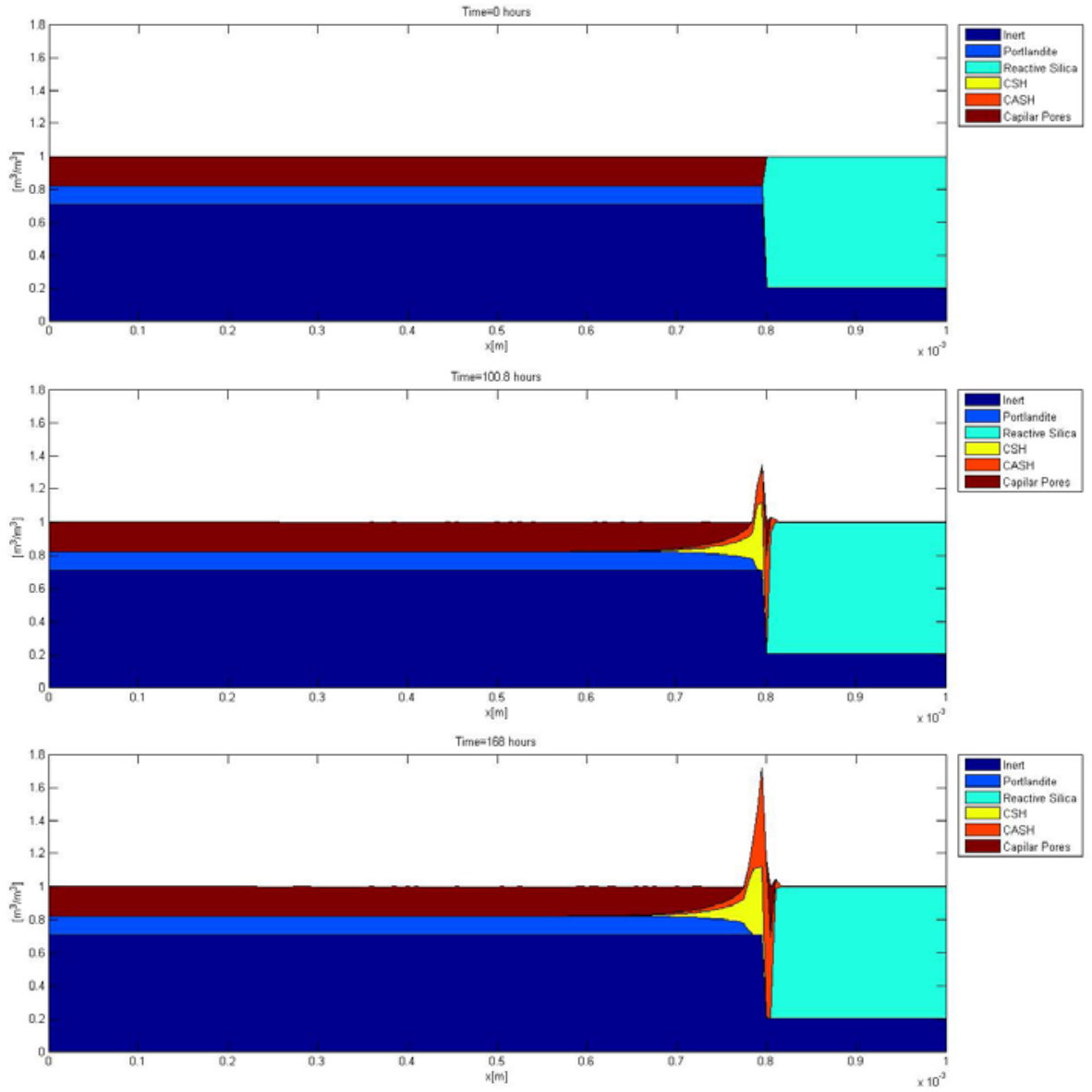

Figure 3: Volumetric solid phase distribution in the interface zone of HCP-glass for different exposure times. Note the gel formation (non-expansive CSH in yellow, and expansive CASH in orange) and the reduction of Portlandite $\left(\mathrm{Ca}(\mathrm{OH})_{2}\right)$ and pore space, near the cement-glass interface. 


\section{CONCRETE SUBJECT TO HIGH TEMPERATURES}

The effects of temperature changes on heterogeneous materials such as concrete, may include internal stresses, cracking and degradation, even if the temperature remains constant thought the specimen cross-section. This is due to the expansion mismatch between components. Those effects are studied by a coupled thermo-mechanical model, where the main material heterogeneities with their detailed (or at least approximate) geometry are represented, and applying to phase the appropriate thermal expansion and material behavior laws. The cause of coupling in this case is given by the thermal boundary conditions as spalling occurs: for the first time step temperature distributions are computed with the boundary conditions applied on the original specimen boundary, but this situation changes as the time goes on and layers of material start dissapearing. Then, the new temperature b.c.s for each time increment are applied onto the new updated domain boundaries.

The example of application presented consists of to a $10 \times 30 \mathrm{~cm}^{2}$ rectangular 2D specimen of concrete subject to prescribed temperature increments on the right vertical edge of the domain only, while thermal flow is assumed null on the other three faces. The displacements normal to the surface are also restrained along those faces. The time-dependent imposed temperature values correspond to the standard curve given in the fire-resistance tests recommendation ISO834-1 which are defined by $T=345 \log _{10}(8 t+1)+20$, where $\mathrm{T}$ is the temperature in ${ }^{\circ} \mathrm{C}$ and $\mathrm{t}$ is the time in minutes. In the numerical analysis, concrete is represented by large aggregates particles of dolomite rock surrounded by a matrix that represents mortar and smaller aggregates. The specimen considered has volume fraction of large aggregates $28 \%$ and maximum aggregate size $14 \mathrm{~mm}$ (average aggregate size $10.4 \mathrm{~mm}$ ). A variable coefficient of thermal expansion has been used for mortar. It has been extracted from a volume expansion vs. temperature curve obtained numerically in (Rodriguez et al., 2011). For the aggregate a constant coefficient of thermal expansion extracted from the experimental results of (Cruz \& Guillen, 1980), who obtained independent expansion curves for aggregates, cement, mortar and concrete, are used.

Figure 4 depicts the temperature distribution (top) and cracking/spalling situation (bottom) obtained for four different calculation times, which correspond to prescribed temperatures of $420,660,680$ y $720^{\circ} \mathrm{C}$, respectively. As already mentioned, the analysis includes the thermo-mechanical coupling due to the evolving geometry and boundary conditions of the thermal action, which, at each stage of the calculation are moved to the new boundary resulting from the mechanical spalling. More details of these calculations, including the extension to 3D, can be found in Perez et al. (2013).

\section{CONCLUDING REMARKS}

The mesomechanical model for concrete previously developed for purely mechanical actions, which is based on Voronoi-generated geometries of the larger aggregates and cracking via fracture-based zero-thickness interface elements, seems to provide a convenient basis for extensions to coupled durability mechanics calculations. Until now, the model has been developed for drying shrinkage, external sufate attack and 
mechanical effects of high temperatures. On-going work devoted to alkali-silica reaction (ASR) seems very promising, with a three-field diffusion-reaction chemical model which has yet to be coupled to the mechanical analysis, but that has already been shown to qualitatively and quantitatively reproduce some experimental measurements of sandwich-type specimens subject to standardized accelerated ASR tests. From the chemical viewpoint, the formulation has the advantage that it incorporates the recently identified role of Calcium in ASR expansions (Leeman et al, 2011 ) , i.e. depending on local conditions (including $\mathrm{pH}$ and $\mathrm{Ca}^{++}$concentration) the model can lead to either generation of non-expansive $\mathrm{CSH}$, expansive $\mathrm{CASH}$, or a mix of both in varying proportion.
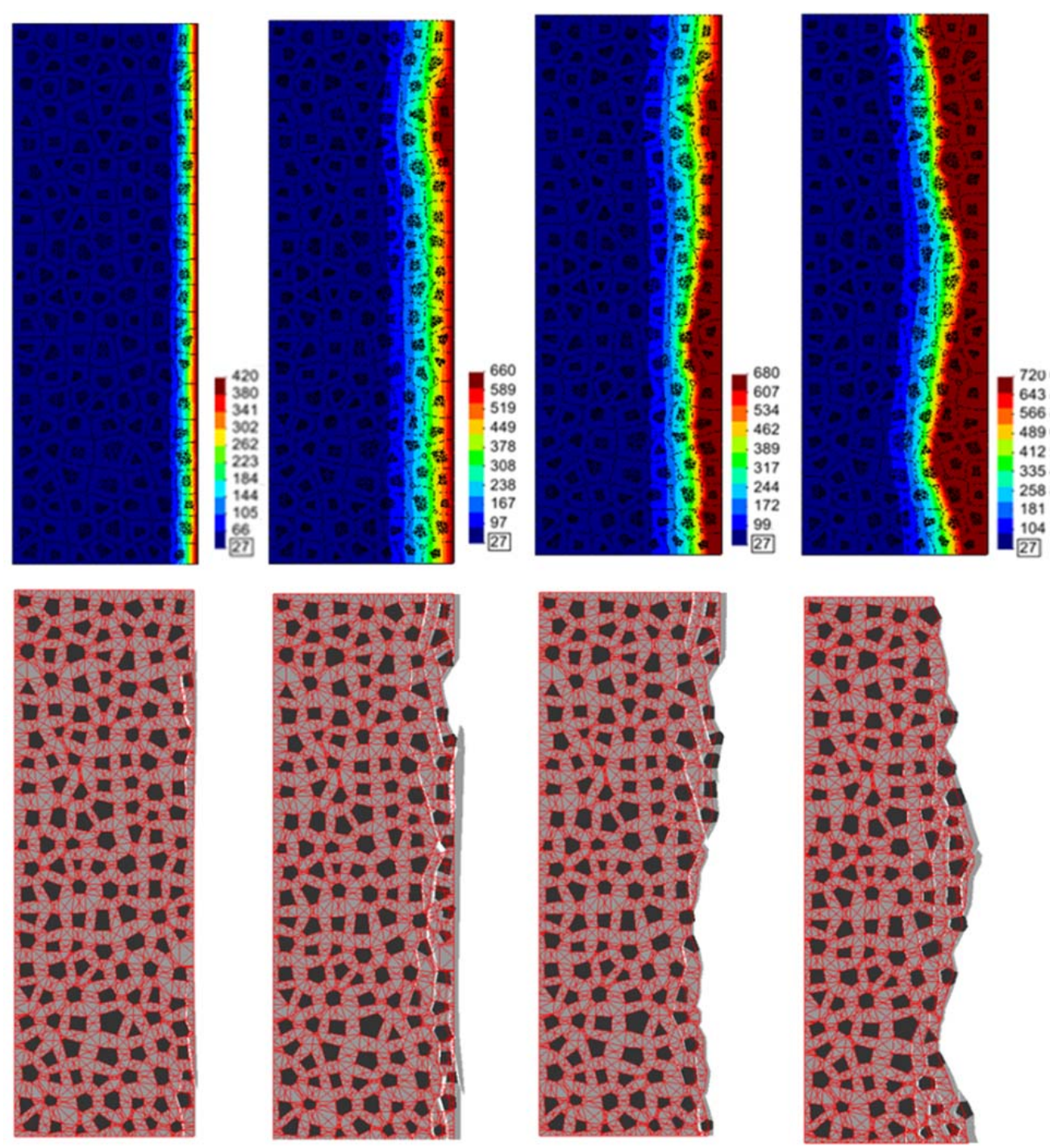

Figure 4: Evolution of temperature and deformation for the prescribed boundary values: $\mathrm{T}=420,660,680$ y $720^{\circ} \mathrm{C}$, corresponding to the ISO curve times: $t=1.68 ; 8.82 ; 10.10$ and 13.23 minutes. 


\section{ACKNOWLEDGEMENTS}

Partial support comes from grants BIA-2012-36898 from MEC (Madrid), including FEDER funds, and 2009SGR-180 from AGAUR-G. de Catalunya (Barcelona). The first author also thanks MICINN (Madrid), for his FPI doctoral fellowship.

\section{REFERENCES}

Caballero, A., Carol, I., López C. M. (2007). "3D mesomechanical analysis of concrete specimens under biaxial loading”. Fatigue and Fracture Engng. Mat. and Structures, 30: 877-886.

Carol, I., Prat, P. C., López, C.M. (1997). A normal/shear cracking model. Application to discrete crack analysis. Engng. Mech. ASCE, 123: 765-773.

Carol, I., López, C.M. and Roa, O. (2001). Micromechanical analysis of quasi-brittle materials using fracture-based interface elements. Int. J. Num. Meth. Engng., 52:193215.

Cruz, C. R., and Gillen, M. (1980). "Thermal expansion of Portland cement paste, mortar and concrete at high temperatures". Fire and materials, 4:66-70

Idiart, A., López, C.M., Carol, I. (2011a). "Modeling of drying shrinkage of concrete specimens at the meso-level”. Materials and structures RILEM, 44: 415-435.

Idiart, A., López, C.M., Carol, I. (2011b). "Chemo-mechanical analysis of concrete cracking and degradation due to external sulfate attack: A meso-scale model”. Cement \& Concrete Composites, 33:411-423.

Leemann, A., Le Saout, G., Winnefeld, F., Rentsch, D., \& Lothenbach, B. (2011). AlkaliSilica Reaction: the Influence of Calcium on Silica Dissolution and the Formation of Reaction Products. J. Am. Ceram. Soc. 94: 1243-1249.

Liaudat, J., López, C.M. and Carol, I. (2013).”Diffusion-reaction model for alkali-silica reaction in concrete". In Proc. COMPLAS XII. E. Oñate, D.R.J. Owen, D. Peric and B. Suárez (Eds). CIMNE (2013) (in press).

López, C.M., Carol, I., and Aguado, A., (2000). Microstructural analysis of concrete fracture using interface elements. In O־nate, E. et al., editors, Proc. ECCOMAS 2000.CIMNE (Barcelona, Spain). ISBN 84-89925-70-4 (in CD-ROM).

López, C.M., Carol, I., and Murcia, J, (2001). Mesostructural modeling of basic creep at various stress levels. In Ulm, F.J., Bǎzant, Z.P., and Wittmann, F.H., editors, Creep and Shrinkage of Concrete Structures, pp. 101-106. John Wiley (New York).

López C.M., Carol I., Aguado A. (2008). "Meso-structural study of concrete fracture using interface elements. I: numerical model and tensile behavior" and "II: compression, biaxial and Brazilian test”. Materials and Structures, 41: 583-620.

Pérez, A., Rodriguez, M., Lopez, C.M. and Carol, I. (2013). “3D Meso-mechanical analysis of cracking and spalling of concrete subject to high temperatures". In Proc. COMPLAS XII. E. Oñate, D.R.J. Owen, D. Peric and B. Suárez (Eds). CIMNE (2013) (in press).

Rodríguez, M., López, C.M., Carol, I., and Murcia, J. (2011). "High temperature effects in mortar and concrete specimens using a meso-mechanical model with fracture based zero-thickness interface elements”. Proc. COMPLAS XI, Barcelona, Spain.

Segura, J. M. and Carol, I. (2004). "On zero-thickness interfase elements for diffusion problems”. International Journal for Numerical and Analytical Methods in geomechanics, 28: 947-962 